\title{
Interaction and Co-located Collaboration in Large Projection-Based Virtual Environments
}

\author{
Andreas Simon ${ }^{1}$, Armin Dressler ${ }^{1}$, \\ Hans-Peter Krüger ${ }^{1}$, Sascha Scholz ${ }^{1}$, and Jürgen Wind ${ }^{2}$ \\ ${ }^{1}$ Fraunhofer IMK Virtual Environments, Sankt Augustin, Germany \\ ansimon@gmail.com \\ \{armin.dressler, hans-peter.krueger, \\ sascha.scholz \} aimk. fraunhofer. de \\ ${ }^{2}$ Vertigo Systems, Köln, Germany \\ juergen.windevertigo-systems.de
}

\begin{abstract}
Conventional interaction in large screen projection-based display systems only allows a "master user" to have full control over the application. We have developed the VRGEO Demonstrator application based on an interaction paradigm that allows multiple users to share large projection-based environment displays for co-located collaboration. Following SDG systems we introduce a collaborative interface based on tracked PDAs and integrate common device metaphors into the interface to improve user's learning experience of the virtual environment system. The introduction of multiple workspaces in a virtual environment allows users to spread out data for analysis making use of the large screen space more effectively. Two extended informal evaluation sessions with application domain experts and demonstrations of the system show that our collaborative interaction paradigm improves the learning experience and interactivity of the virtual environment.
\end{abstract}

\section{Introduction}

Since the introduction of the CAVE [4] over ten years ago, large, projection-based stereoscopic displays have become a commodity item. Wide-screen stereoscopic walls, CAVEs or even bigger theatre-like installations like the i-Cone [18] are an established part of the infrastructure for 3D graphics visualization, not only at research labs and universities, but also in large corporations, in particular in the automotive and in the oil-and-gas industry. Although these systems are large, expensive and difficult to maintain, they have eclipsed the use of small, inexpensive, personal head mounted displays (HMDs) in all but a few application areas. In part this is due to the fact that they are large, single screen displays, allowing multiple users to directly view and share the experience of a virtual environment in a group; all this without the immediate need to make changes to the software or hardware.

The use of head mounted displays for doing real work in a virtual environment certainly is an acquired taste. We would argue that user preference for projection-based displays over HMDs is not just influenced by display quality, but is motivated by collaboration aspects and the learning experience for new or casual users. First-time 
users of HMDs get to wear a heavy helmet (smaller, still obtrusive Sony Glasstronstyle goggles have a narrow field of view and no stereoscopic viewing), isolating them from their familiar environment and from other people. It is difficult for a demonstrator to teach and guide a new user, since it is hard to know exactly what she really sees and does. For projection-based display systems, instead of experimenting on their own, new users typically join an experienced demonstrator who is guiding them through the environment. At some point the demonstrator may carefully hand over controls, remaining alert to immediately help whenever the learner gets lost. Unfortunately this is where the story typically ends. Although in a projection-based system a group of viewers can share the experience of the virtual environment, in current applications only one user can interact and control the application at a time.

In order to correctly match real and virtual space and to achieve fully correct spatial viewing, the projection of a stereoscopic image must match the exact location of the viewer. This image has to be continuously updated to the viewer's current viewing position and orientation. Since practically all display systems are only capable of projecting a single stereoscopic image, only the one head-tracked user in a display sees a correct spatial image. Other participants in the same display share this view, leading, from the individual user's perspective, to parallax: distortion and a mismatch between the real and the perceived virtual space.

The single head-tracked user in a display is often called the "master user" of the application, and operates specialized interaction devices that are unfamiliar and usually hard to learn. All other participants are practically only looking over the shoulder of the master user, without tools to interact on their own. In typical theatre-like demonstration centers, the master user even sits at a desk outside the display, steering the application from a conventional desktop interface. In this case, the "interface" operates by viewers inside the virtual environment asking the master user at the keyboard to change parameters in the application.

Recently, we have presented two rendering techniques-omnistereo projection [19] and multi-viewpoint images [20] - that allow the projection of different image elements with different perspectives in a single, consistent, stereoscopic image. This allows displaying virtual interaction elements in each user's perspective, correctly aligning real devices and their virtual representations to overcome the parallax problem for multi-user interaction.

Based on the concept of Single Display Groupware (SDG) systems [22][13], we develop a new interaction paradigm for co-located collaboration in large projectionbased virtual environments. We apply this concept to the VRGEO Demonstrator, an application for the review of volumetric data sets in the oil-and-gas industry. Following Buxton et. al [3], the overriding issue for the successful use of large displays is ultimately a story about interaction, not displays. For the system to be of value, viewers must be able to create, manipulate, explore, and annotate in the environment. Key goals for the development of our interaction paradigm for projection-based virtual environments are to improve the level of interactivity and the learning experience by introducing a co-located collaborative interface and using common device metaphors in a virtual environment; also we want to exploit the screen space for large virtual environment displays better by introducing multiple workspaces that can be arranged to structure the display volume. 
The remainder of this paper is organized as follows: Section 2 introduces techniques to support co-located collaboration in a projection based virtual environment. Sections 3 and 4 present spatial interaction techniques with a 3D tracked PDA and the PDA GUI interface; section 5 discusses related work. In section 6 we present experiences from trials and demonstrations with the VRGEO Demonstrator. Finally, section 7 presents conclusions and discusses opportunities for future work.

\section{Co-located Collaboration}

We introduce three techniques to support co-located collaboration. Multi-viewpoint images solve the parallax problem for direct interaction with multiple users in a panoramic projection-based display. Multiple workspaces-in the case of the VRGEO Demonstrator boxes containing geoscientific volumetric data sets-allow users to spread out the data over the whole display and make better user of the large display surface. Finally, we introduce PDAs into the interface, to implement a common private interface for each user.

\subsection{Multi-viewpoint Images}

We use multi-viewpoint images [20], composed out of different image elements projected from multiple viewpoints, to overcome the parallax problem in non-headtracked applications and to enable multi-user interaction in the i-Cone projectionbased display.
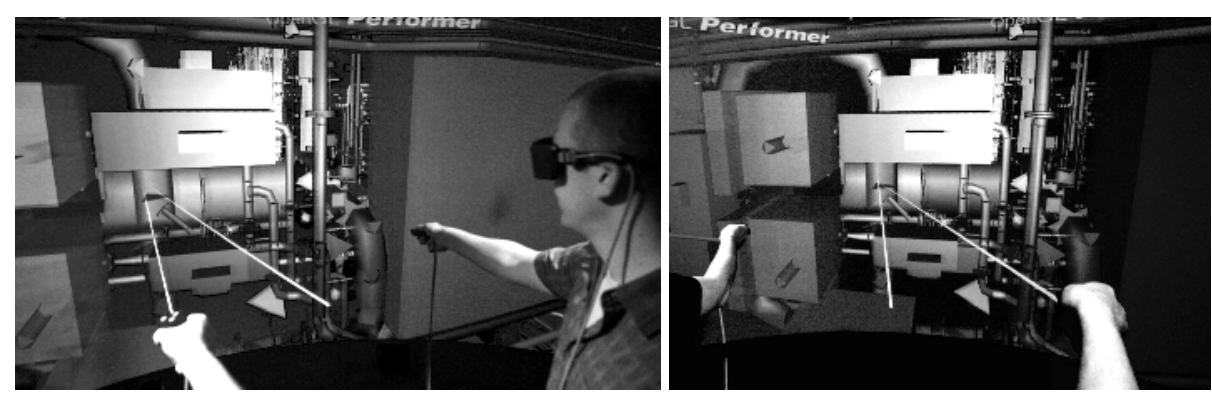

Fig. 1. Left user's vs. right user's view of a multi-viewpoint image: Picking rays align correctly from the respective user's viewpoint

The multi-viewpoint image in Figure 1 is one and the same image. It combines three different viewpoint projections: One for the main scene and one for each of the two users. The main scene, containing engines and pipes, is rendered without head tracking from a static viewpoint centered in the middle of the display. For each of the two users, the user's picking ray is rendered from the respective user's head-tracked viewpoint. This places the picking ray, seen from that user's perspective, in correct alignment with his tracked interaction device. 


\subsection{Multiple Workspaces}

Conventionally, projection-based virtual environments displays like the CAVE are used with a single active scene and a single focus of attention. There are no simultaneous, competing applications, the application complexity is typically quite low, and there is no notion of spatial dividers and of separate workspaces, since there is only one "master user" equipped with interaction devices.

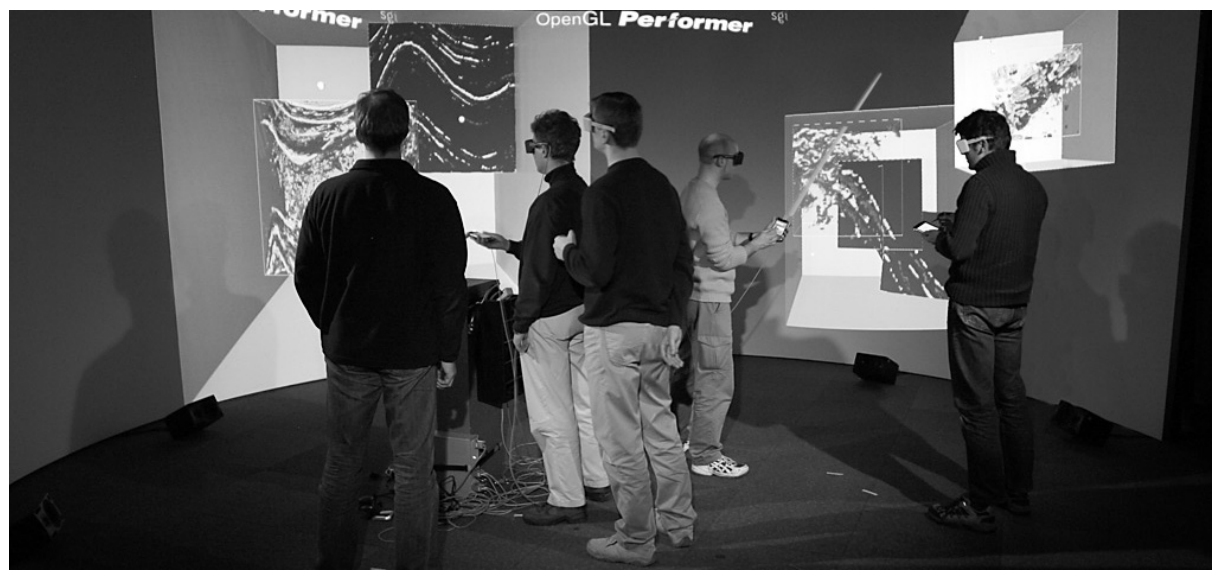

Fig. 2. A group of users working with multiple workspaces in a $240^{\circ}$ i-Cone ${ }^{\mathrm{TM}}$ display

Large display surfaces are essential for supporting collaborative, or even individual activities [9] because they allow users to simultaneously spread out and arrange several data items. In our multi-user paradigm, we have introduced the concept of multiple work areas in a virtual environment, allowing users to work with multiple 3D data sets side by side, but also allowing them to split into subgroups or work on specific problems independently of each other (Figure 2). In the VRGEO Demonstrator, we use boxes-separate 3D workspaces—each containing one 3D volumetric data set. Inside a box visualization tools like volumetric rendering lenses or texture slices allow to view and analyze different aspects of the data set, set markers and take snapshots. The boxes work as spatial separators and allow users to arrange and partition different visualizations. They are a spatial analogue to windows in a conventional 2D interface and allow users to easily grab a coherent part of the scene and move it next to another for comparison.

By introducing workspace boxes, we establish multiple foci of work in a panoramic environment. Users use this by forming different work areas and alternating between different solutions, using the large screen area for direct comparison and as a visual memory. Alternatively, the large screen and the spatially distinct work areas allow a larger group to split up temporarily to analyze different sub-problems, enabling users to alternate between collaboration and individual work and preparation. The boxes also form a clear visual background and separation for the individual data sets, avoiding confusion, and allow users to easily layout a spatial arrangement of data sets around them. 


\subsection{Public vs. Private Display}

Introducing PDAs into a large immersive projection display as an additional individual and private display for each user, introduces the separation of public and private data into our virtual environment (Figure 3). It also solves the problem of separating the representation of the application state and the individual contexts and modes for each user [13] by allowing us to put all the individual application state information on each user's PDA interface.

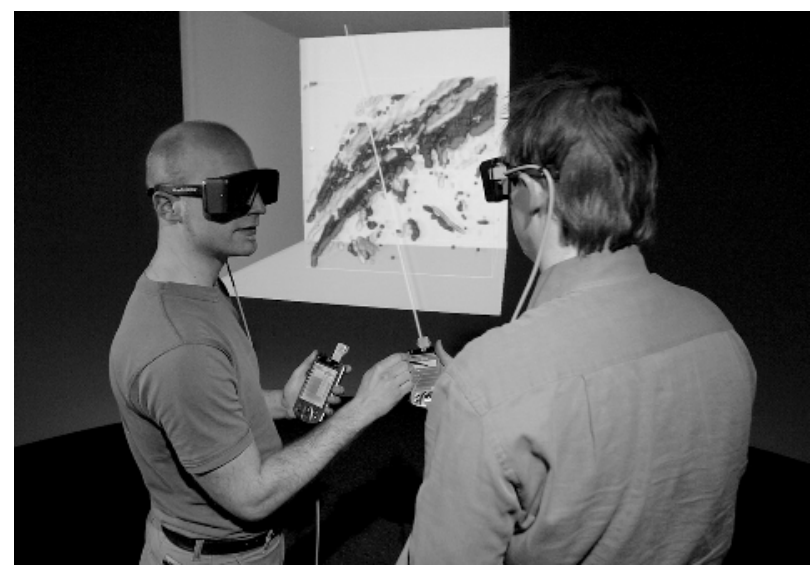

Fig. 3. Teaching the interface in a collaborative environment

In some situations, for example reviewing numerical or textual data on the PDA or to follow the interaction of a user, we would like to share the information on another user's PDA directly. We have implemented a function to explicitly share the state and "jump" to the interface pane of another user's PDA, joining the private interfaces by connecting both PDA interfaces (Figure 4). When a user jumps to the interface of a colleague, he will share the state and display of the other PDA's GUI. In shared mode, both PDAs will behave exactly the same, if one user is changing a value with a slider, the other user's slider will move simultaneously to the same value. Both users stay connected (when one user changes a pane or selects an object, the other PDA follows) until one of them explicitly disconnects.
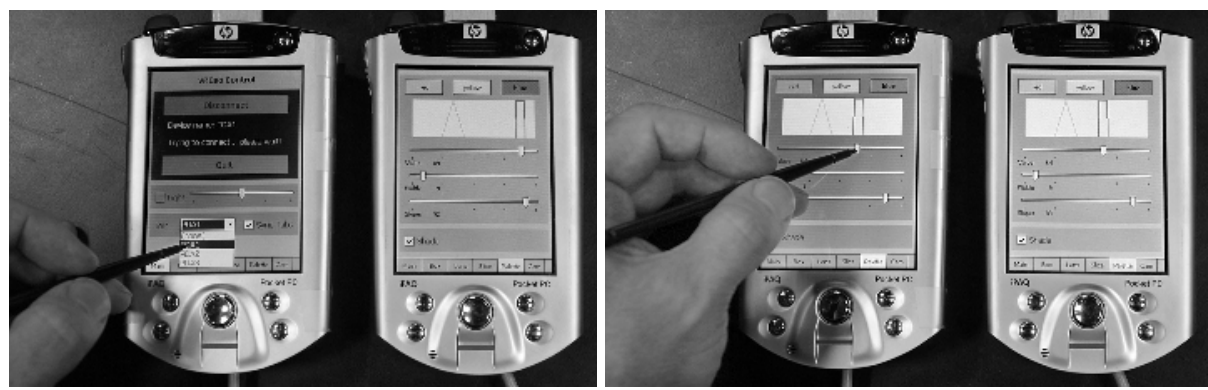

Fig. 4. Connecting (left) and sharing (right) two PDA interfaces 
Joining the PDA GUIs allows transparency for other users for the manipulation of complex interfaces on the PDA (temporarily sharing the private interface) and allows users to very effectively teach each other the application.

\section{3D PDA Interface}

Tracking of 3D position and orientation of the PDA as part of the spatial interface enables us to integrate the device as a functional prop into the three-dimensional virtual world. This allows us to relate to interface metaphors of common devices in the real world, making the interface accessible to new and infrequent users of virtual environments.

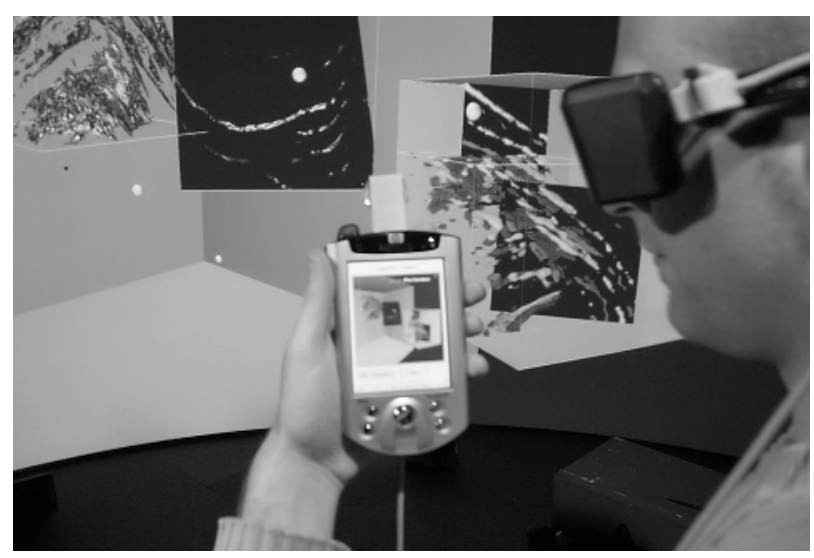

Fig. 5. Taking a snapshot using the PDA like a real camera in the virtual environment

\subsection{Snapshot Camera}

The use of a tracked PDA as a virtual environment display device of its own was proposed by Fitzmaurice [6], who has investigated the use of Chameleons. Chameleons are small-screen devices that provide a small but mobile window to a virtual world behind the screen. The position and orientation of the device is used to determine the view-frustum into the virtual scene. In our application, we use the same technique to render an image to the PDA, using it as a virtual camera to provide a natural and direct interface to take snapshots of the virtual environment. The PDA screen acts as the finder, reacting to the orientation and position of the PDA in the same way as a real camera would (Figure 5). As with a real camera, the user can frame the image, zoom and take a snapshot by pressing a button. The resulting image is transmitted and stored locally on the file system of the PDA, providing the user with a personal copy.

\subsection{Virtual Light}

In similar fashion to using PDA's display as a camera finder, we use the backlight of the screen to act as an interface to a moving virtual light source. For rendering, a 
directional light with a $180^{\circ}$ light cone is attached to the position and orientation of the tracked PDA, facing the same direction as the light cone of the PDA screen's real backlight. In order to highlight some close-up object, the user turns his PDA aroundwith the screen facing into the scene-and shines virtual light onto the rendered scene. This interaction produces a very strong illusion and suspension of disbelief [5], since the backlight on the PDA acts on real objects (e.g. the users hand) in the same way as the virtual light source acts on virtual objects.

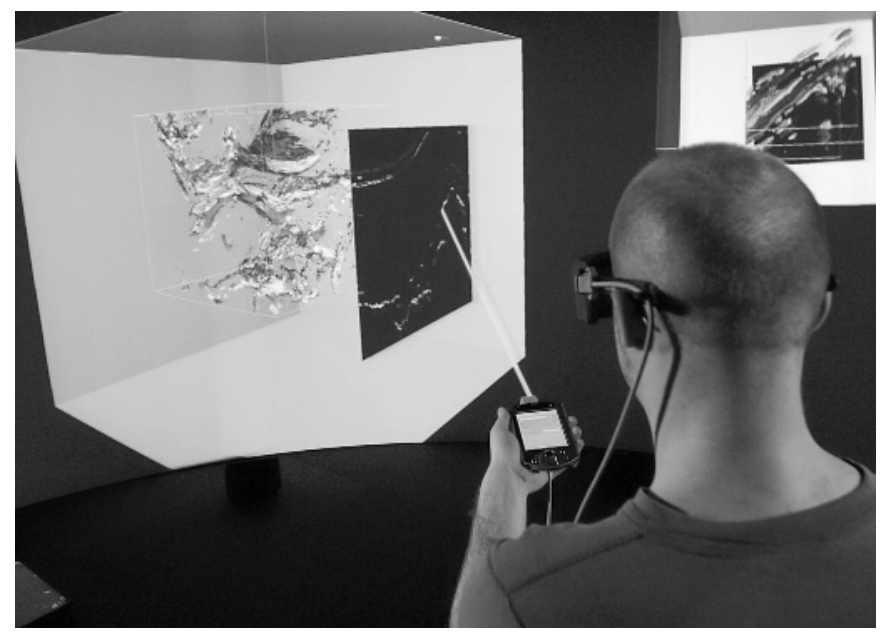

Fig. 6. Using the PDA as a virtual laser pointer for object selection

\subsection{Laser Pointer}

The tracked PDA is also used as a pointing device for 3D object selection by ray casting [10], extending a virtual lightsaber [11] from the tip of the PDA. For selection, the user points the lightsaber at a virtual object (Figure 6) and clicks the top-left PDA button. This $3 \mathrm{D}$ object selection will also set the state of the PDA GUI and places the corresponding 2D interface pane on the PDA on top. Laser pointing is also a common device metaphor from the real world-digital projector remote controls typically incorporate real laser pointers in a similar fashion.

\subsection{Scaled Grab Motion}

We want to place objects at a comfortable viewing distance and spread them out over a large field of view; therefore, users need to be able to perform interaction and object motion at a distance in an effective way. In a collaborative environment, we cannot use travel to move larger distances inside the virtual environment, since this would disturb other users-similar to collaboratively browsing a rotating postcard standinstead, we have to be able to select (grab) distant objects and pull them close or push them back with minimum effort. 

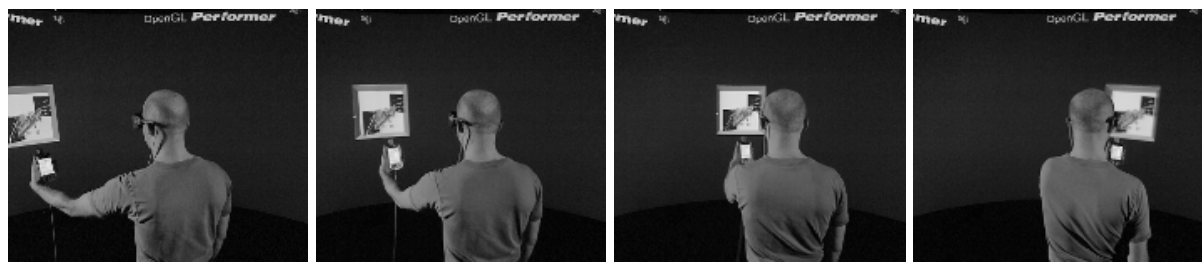

Fig. 7. Large object motion with Scaled Grab: Note alignment between PDA and virtual object

Selecting an object by ray casting with the PDAs Laser Pointer automatically places a pivot point at the intersection of the lightsaber and the object's surface. After selection, a user can drag the selected object, holding the top-left PDA button and moving the PDA.

For effective dragging and moving objects over large distances, we have developed a virtual motion technique we call Scaled Grab. Scaled Grab combines image plane selection and motion techniques and is similar to world-in-miniature (WIM) object manipulation [23]. Unlike Mine's Scaled-world Grab [12], which scales down the world to bring the selected object within reach of the user, Scaled Grab scales up the users range of hand motion, to extend to the selected object (Figure 8). In this respect it behaves like a WIM, but without introducing an explicit miniature representation of the object. Scaled Grab rather uses the PDA as a handle on the selected object instead.

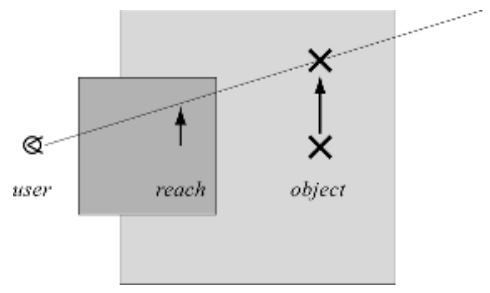

Fig. 8. Scaled Grab to extend the user's reach

The distance of the selected pivot point on the object's surface to the user's eye point determines the scale-ratio for hand to object motion. This ensures constant alignment in the image plane of the virtual object's motion with the tracked point on the PDA. Note that in Figure 7 the tip of the PDA and the workspace box on the screen remain aligned in the image, although the box is about $3 \mathrm{~m}$ away from the user. Also, using this technique, the ratio between the subtended angle-the relative size in the image plane - of the PDA and the dragged object remains constant. This behavior gives very good and consistent feedback of the synchronized motion between object and handle (PDA) to the user.

Rotation of virtual objects presents a challenge, since rotation with a far away center of rotation can result in large, unwanted motion of the object. This is known to lead to confusion since the object can rotate out of the field of view and magically disappear. With Scaled Grab we use the pivot point that the user has placed by selecting the object as center of rotation and rotate the virtual object around a meaningful, user-defined center. 


\section{PDA GUI Design}

The primary motivation for introducing PDAs as an interface into our collaborative virtual environment is the same as for Myers et. al [13] who have introduced PDAs into single display groupware (SDG) systems: The PDA as personal device allows us take advantage of the fact that users are familiar with the device and have already learned the interface paradigm outside of our environment. This reduces barriers for new or infrequent users to join a team.

When using a PDA-based GUI interface in a virtual environment, we have to consider a number of issues that influence the design of the PDA interaction. Major issues concern the viewing of the PDA screen. Shoemaker [17] has noted that using a PDA display forces a rapid change in the focus of attention over different displays and over a wide depth range, when a user is manipulating or reading something on the PDA screen in his hand and has to look back into the environment to see the result. In our case this environment even consists of a stereoscopic virtual image on a screen. Stereo glasses - needed for stereoscopic viewing of the projected images-further reduce the contrast and readability of the PDA's screen.
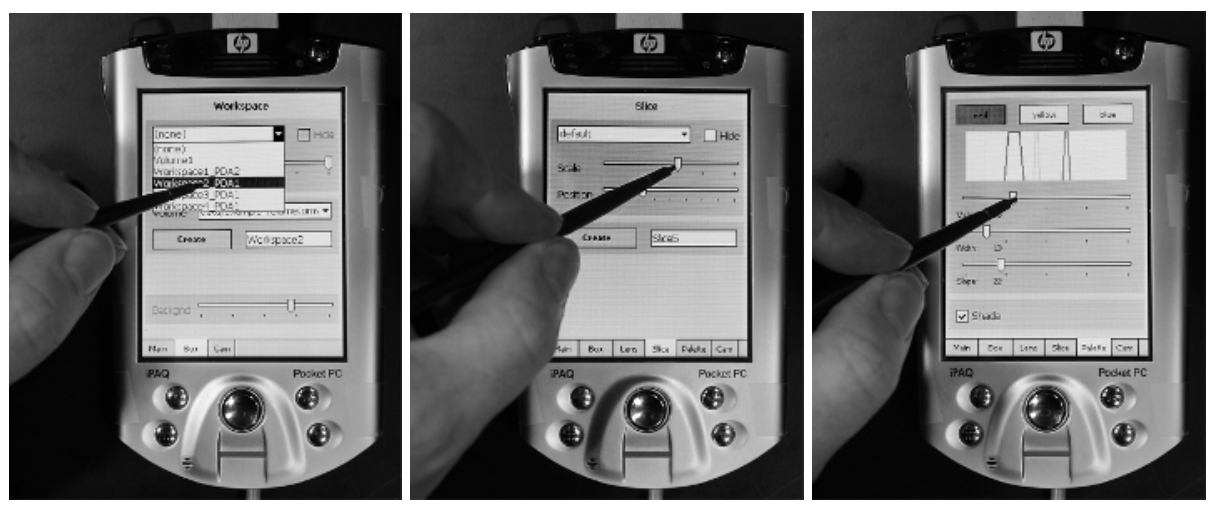

Fig. 9. PDA GUI organized in simple hierarchically ordered panes

Another issue concerning the design of the PDA GUI is the possible disruption of context in the interface. The 3D ray-casting object selection allows the selection of objects by pointing directly at them in the virtual environment. Displaying the corresponding PDA interface for the selected object (just as if the user had selected this object with the GUI on the PDA) produces a jump in context on the PDA that may be unexpected to the user, since she is not looking at the PDA while performing the selection.

With the concerns for readability and the need for a simple, clear design to enable the user to follow external context switches as a result of the $3 \mathrm{D}$ selection interface, we have structured the PDA GUI in simple, static panes that are selected through tabs. Only a single tab set, with tabs aligned with the lower edge of the screen, is used (Figure 9). An additional bold headline on top of each pane indicates the name of the currently selected pane and makes external context switches better visible. Tabs order 
the panes in a sorted, hierarchical fashion that corresponds to the object relationships in the application: After creating a new workspace Box, panes for Lens, Slice and Palette open up and are ordered directly after the Box tab of the workspace pane.

\section{Related Work}

Stewart et al. [22] have coined the term Single Display Groupware (SDG). Stewart's KidPad [21] is a SDG environment for kids, where multiple mice are connected to a single computer. It uses "local tools" on Pad++, a drawing program, where each tool does exactly one thing. Background studies showed that children often argue and fight when trying to share a single mouse, but when using separate mice, cooperated more effectively. The Pebbles project by [13] connects multiple PDAs to a main computer in a SDG scenario. In the applications, the PDAs are primarily used to control multiple mouse and keyboard input to whiteboard applications. Greenberg has studied the role of public and private information in a SDG application with PDAs [7]. In this system, mobile individuals carry PDAs and can create personal notes at any time. When these individuals gather in a meeting, they can selectively publicize these notes by transferring them to the shared display. Rekimoto has developed a similar system involving a shared display and private mobile devices [18]. Rekimoto introduces mobile computers and PDAs as common, spatially tracked interaction devices into his shared environment. With this system, a PDA is used as a tool palette and as a data entry palette. At any time a user can "pick and drop" private information from the PDA with a special stylus and place it on the shared, public display.

Most of the work on co-located collaboration in virtual environments focuses on head mounted displays (HMDs) since they are inherently suitable for multi-user display. Studierstube [24] was one of the first systems to show the potential of AR for co-located collaboration. [14] describes an HMD based Augmented Reality (AR) system which allows multiple participants to interact with two- and three-dimensional data using tangible user interfaces. The system is based on a tabletop metaphor and uses camera-tracked markers on paper cards or props to provide a tangible interface to virtual objects. As in [15] PDAs are used as a data entry palette, using "pick and drop" to drag virtual objects onto the table.

Only a few multi-view projection-based displays, to allow multiple users to interact and collaborate in a virtual environment sharing a common viewspace, have been developed. These systems allow the display of more than one stereoscopic image, displaying individual perspective views for each user to overcome the parallax problem. The duo-responsive workbench [1] is a multi-view display system that supports two users by sequentially displaying four images on the screen of a responsive workbench. Both users wear tracked data-gloves and use direct manipulation techniques to select, grab and move objects. A static menu, shared by both participants is attached to the edge of the tabletop surface. Other multi-view projection displays use a spatial barrier to achieve multiple views in a shared view-volume, but on different screens. The PIT [2] consists of an L-shaped arrangement of two screens, with each user looking at one of the screens. With the Illusion Hole [8], a view barrier separates the different views of users standing around a small hole over a workbench, each user 
looking through the hole at a different part of the workbench screen. Both papers concentrate on the technical aspects of the displays and do not discuss interaction or collaboration.

It is interesting to note, that previous virtual environment scenarios deal exclusively with outside-in viewing in round table situations and concentrate on the use of direct manipulation interfaces. We use the PDA GUI interface independently and concurrently to a 3D interface on the shared screen, combining PDA meeting style applications with a multi cursor SDG application. This is a more complex paradigm than PEBBLES [13], similar to the situation of [15] who in turn primarily connects devices through data, and not through their interface. For our application, we combine two very different application and interaction paradigms-3D spatially tracked vs. PDA GUI-in a single environment.

\section{Experiences and Observations}

We have presented the VRGEO Demonstrator on numerous occasions to groups of three to eight visitors. In two 60 minute evaluation sessions, four members of the VRGEO consortium, representing several mayor oil companies, have used the demonstrator. These evaluations sessions have retuned the most valuable feedback.

In the current set up, because of limitations with the Polhemus Fastrack tracking system, we use two fully tracked PDAs, and one additional non-tracked PDA.

There is practically no need to explain the interface of the application at all. Most visitors would grab the PDA and immediately start exploring the interface on their own. We would only explain the use of the top-left PDA button as the select/execute button and demonstrate the conceptually more complex "joining" of two PDAs.

As expected, learning of a new interface in a co-located environment is much more relaxed than in a single user environment. New users would take their time to look and browse the interface, not feeling rushed even in a demo situation. We would frequently observe users discussing functionality with each other. Sharing of the PDA interface through the "jump" function has been effective for teaching, since it allows two users to closely follow each other's actions. Test users liked this function a lot, but report minor problems: they would assume that they were connected when they were not, completely missing the other user's actions. Connect and disconnect functions, placed on the Main pane, are currently too slow to "jump" to the neighbors PDA GUI just to have a peek.

With the introduction of separate workspaces that can be spread out in the display, we have seen that users make much better use of the large screen space, and tend to spread out various boxes over the whole field of view. The ability for a single user to separate a part of the visualization, adjust the viewing parameters to clearly bring out and mark some detail, and quickly rejoin the discussion, changes the possible work flow in this type of application. Tedious adjustments do not have to be performed while the whole group is watching.

In our evaluation scenarios, it was difficult to actually observe true active collaborative behavior. With the oil-and-gas experts we could see that while one user was moving and turning the data set around, another would adjust the color palette of the same volume to segment out new structures. With non-experts we would observe 
more individual viewing of the data and exploration of the interface and less interaction. Occasionally users would "steal" workspace boxes from each other.

The Scaled Grab technique has proven to be very effective and completely transparent to the users. We did not receive any negative feedback on this technique; most users were completely unaware that there was something special going on until we switched the scaling off.

Most users would handle the PDA in their non-dominating hand, to be able to use the PDA GUI with the pen in a normal fashion. For some users this would lead to problems with the 3D PDA interface since they had to handle ray-based object selection and Scaled Grab motion with their non-dominating hand. Although we have not seen severe problems with this issue, the interface seems to favor ambidextrous users.

Overall, using the i-Cone in a collaborative fashion delivers a very different experience than the conventional single user paradigm. Feedback about this was enthusiastic. Although the ergonomics are difficult (handedness problems, tethered tracking, problematic button placement on the iPAQs) the overall effect of introducing the PDA interface into the virtual environment has been very positive.

\section{Conclusions and Future Work}

We have introduced an interaction paradigm for co-located collaboration in large projection-based display systems. Based on the concept of SDG systems, it introduces PDAs as personal interface for users in a virtual environment. Informal observations show that the introduction of co-located collaboration improves the overall user experience and interactivity of the virtual environment. Despite some ergonomic problems with the use of the tracked PDAs, the introduction of common devices and common device metaphors, together with sharing a common interface in a co-located application environment, seems to have a very positive effect on the learning experience of new and casual users.

In the future we will use a wireless optical tracking system, allowing us get rid of all the wires and to support a larger number of active users. With a clip-on mechanism for the optical tracking target, users would be able to bring their own PDAs into a virtual environment session. We would like to develop a more complex application scenario based on our interaction paradigm that encourages more immediate collaboration between users on a demanding collaborative planning and design task.

\section{References}

1. Agrawala, M., Beers, A., Fröhlich, B., Hanrahan, P., McDowall, I., and Bolas, M.: The Two-user Responsive Workbench: Support for Collaboration through Individual Views of a Shared Space. Proc SIGGRAPH'97. ACM Press (1997) 327-332

2. Arthur, K., Preston, T., Taylor, R., Brooks, F., Whitton, M., and Wright, W.: Designing and Building the PIT: A Head-Tracked Stereo Workspace for Two Users. Proc. 2nd International Immersive Projection Technology Workshop (1998)

3. Buxton, W., Fitzmaurice, G., Balakrishnan, R., and Kurtenbach, G.: Large Displays in Automotive Design. IEEE Computer Graphics and Applications (2000) 68-75 
4. Cruz-Neira, C., Sandin, D., DeFanti, T., Kenyon, R., and Hart, J. The CAVE Audio-Visual Environment. ACM Trans. on Graphics 35,1 (1992) 65-72

5. Coleridge, S. (1817). Willing Suspension of Disbelief. Samuel Taylor Coleridge, ed. Jackson, H. (1985) ch 14, 314

6. Fitzmaurice, G. Situated Information Spaces and Spatially Aware Palmtop Computers. Communications of the ACM 36,7 (1993) 38-49

7. Greenberg, S., Boyle, M. and LaBerge, J.: PDAs and Shared Public Displays: Making Personal Information Public, and Public Information Personal. Personal Technologies, 3, 1 (1999)

8. Kitamura, Y., Konishi, T., Yamamoto, S., and Kishino, F.: Interactive Stereoscopic Display for Three or More Users. Proc SIGGRAPH 2001. ACM Press (2001) 231-240

9. Lange, B., Jones, M., and Meyers, J. Insight Lab: An immersive team environment linking paper, displays, and data. Proc CHI'98 ACM Press (1998) 550-557

10. Liang, J., and Green, M. JDCAD: A highly interactive 3D modeling system. Computers \& Graphics, 18,4 (1994) 499-506

11. Lucas, G. Star Wars. Motion Picture (1977)

12. Mine, M., Brooks, F., and Sequin, C. Moving Objects in Space: Exploiting Proprioception in Virtual Environment Interaction. Proc Siggraph'97 (1997)

13. Myers, B., Stiel, H., and Gargiulo, R.: Collaborations using multiple PDAs connected to a PC. Proc ACM CSCW'98. ACM Press (1998) 285-294

14. Regenbrecht, H., and Wagner, M.: Interaction in a collaborative augmented reality environment. Proc CHI 2002. ACM Press (2002) 504-505

15. Rekimoto, J.: Pick-and-Drop: A Direct Manipulation Technique for Multiple Computer Environments. Proc ACM UISF'97. ACM Press (1997) 31-39

16. Rekimoto, J.: A Multiple Device Approach for Supporting Whiteboard-based Interactions. Proc CHI'98. ACM Press (1998) 18-23

17. Shoemaker, G.: Supporting Private Information on Public Displays. Proc CHI 2000. ACM Press (2000) 349-350

18. Simon, A., and Göbel, M. The i-Cone ${ }^{\mathrm{TM}}$ - A Panoramic Display System for Virtual Environments. Pacific Graphics '02 (2002) 3-7

19. Simon, A., Smith, R., and Pawlicki, R. OmniStereo for Panoramic Virtual Environment Display Systems. Proc IEEE Virtual Reality'04 (2004) 67-73

20. Simon, A., Scholz, S. Multi-Viewpoint Images for Multi-User Interaction. Proc IEEE Virtual Reality'05 (2005)

21. Stewart, J., et al.: When Two Hands Are Better Than One: Enhancing Collaboration Using Single Display Groupware. Proc SIGCHI'98. ACM Press (1998) 287-288

22. Stewart, J., Bederson, B. and Druin, A.: Single Display Groupware: A Model for Copresent Collaboration. Proc ACM CHI'99. ACM Press (1999) 286-293

23. Stoakley, R., Conway, M., Pausch, R. Virtual Reality on a WIM: Interactive Worlds in Miniature. CHI'95 (1995) 265-272

24. Szalavari, Z., Schmalstieg, D., Fuhrmann, A., and Gervautz, M. Studierstube: An Environment for Collaboration in Augmented Reality. Virtual Reality, 3-1 (1998) pp. $37-48$ 